\title{
LE PAROLE CHIAVE DELLA RIVOLUZIONE TECNOLOGICA E LE NUOVE TENDENZE LESSICALI
}

Snježana Bralić, Filozofski fakultet Sveučilišta u Splitu, sbralic@ffst.hr

$10.31902 /$ fll. 30.2020 .9

UDK 811.131.1'373.4

Tenendo presente che il progresso tecnologico ha invaso ogni ambito della nostra vita, questo intervento servirà a capire come le innovazioni tecnologiche, e il linguaggio cui danno vita, non siano più un settore o un gergo specialistici, ma un modo del vivere e del parlare contemporanei. Si tratta di una riflessione non solo linguistica in cui le parole e le espressioni nuove, formatesi come risultato del progresso e della rivoluzione tecnologica, costituiscono una ricchezza lessicale dell'italiano, tratta da quotidiani, fonti lessicografiche e siti internet. Dalla possibilità di far comunicare strumenti diversi o di racchiudere in un unico strumento funzioni finora disperse in più apparecchi, sono nati i termini che indicano l'orizzonte privilegiato della tecnologia moderna: multifunzione, compatibilità e interconnessione.

Nell'italiano, che prima di adattarsi al mondo digitale ha ricavato dall'inglese la maggior parte dei termini legati alla tecnologia, rimangono ancora molte oscillazioni terminologiche. Secondo le parole di Gino Roncaglia "non si tratta semplicemente di analizzare prestiti e neologismi di un settore 'alla moda', ma di comprendere il funzionamento e i cambiamenti della lingua nel suo incontro con un ambiente comunicativo nuovo". Quindi si pone la domanda se la tecnologia parla, se ha cambiato la lingua o vi ha aggiunto semplicemente un lessico che abbonda di sigle e anglismi, un linguaggio nuovo che rispetta le regole della velocità e della sintesi. Molti termini strettamente tecnologici, sentiti come ostici, suscitano resistenza nei singoli parlanti, tanto che si parla di distinzione fra tecnofoni e tecnoanalfabeti, cioè tra quelli che sanno quali nomi dare alle trasformazioni quotidiane e quelli che le usano, senza riuscire a nominarle. Ci riferiamo al fenomeno della diffusione di nuove forme, di veri e propri internazionalismi che si adattano o ricalcano in vario modo, elementi lessicali di origine inglese o angloamericana come in tanti altri settori.

Parole chiave: lessico italiano, comunicazione digitale, linguaggio tecnologico, morfologia lessicale, neoformazioni, parole alla moda, prestiti, sigle, termini informatici 


\section{La tecnologia condiziona la nostra vita e il nostro parlare quotidiano}

Considerando il fatto che il progresso tecnologico ha invaso ogni ambito della nostra vita individuale e collettiva, stravolgendone la fisionomia e accelerandone i ritmi, con questo intervento si cerca di riflettere sulle innovazioni tecnologiche e sul linguaggio cui danno vita, attraverso il nostro vivere e il nostro parlare quotidiano. La realtà ci fa muovere in ambiti e sistemi di regole che semplicemente non avevamo previsto e che nessuno di noi domina bene e fino in fondo (Granieri 5). Perciò la nostra è una riflessione non solo linguistica, ma anche tecnologica, nella quale i neologismi rappresentano una ricchezza lessicale tratta da quotidiani italiani, fonti lessicografiche e siti internet.

I nostri rapporti con gli strumenti nuovi, senza i quali le nostre aspettative e le nostre possibilità sarebbero molto ridotte, entrano oggi in una fase completamente nuova. II mondo cambia e si "ricomplica" ogni giorno. La parola chiave, probabilmente, è "accelerazione" (Granieri 6). La tecnologia, dunque, condiziona la nostra vita e anche il nostro modo di parlare cercando, da un lato, di espandersi fino a coprire ogni ambito della vita quotidiana, dall'altro miniaturizzarsi. Per questo i prefissi diminutivi sono le sue formule preferite. Mentre il mercato combatte la sua battaglia a colpi di mega e di iper, il progresso tecnologico ha scelto come proprio indice la piccolezza: minidisc, minirobot, minitelecamera, microprocessori, microcellulare, microrobot, nanotecnologia, nanocomputer, nanochirurgia, nanomateriale, nanomedicina, nanoscienza (Adamo e Della Valle 2009, 674-675). Inoltre, il progresso tecnologico del XXI secolo ha creato una tecnologia pervasiva ma non invasiva, una tecnologia che è sempre a portata di mano (basta sfiorare un touch screen, 'uno schermo sensibile al tocco') e in grado non solo di soddisfare i nostri desideri, ma di anticiparli, come i software agents, che sanno quello che l'utente vuole prima ancora che la volontà sia arrivata dalla mente alla mano (Galli 37).

Si pone la domanda se la tecnologia parla, se ha cambiato la lingua o vi ha aggiunto semplicemente un arido lessico fatto di sigle e anglismi inespugnabili. Da una parte, saper digitare non equivale a saper scrivere perché l'italiano digitato è una varietà diversa dall'italiano scritto tradizionalmente inteso (Serianni 25). E dall'altra il confronto con le formazioni lessicali del linguaggio di internet, in effetti, è opprimente perché i termini strettamente tecnologici sono ostici, fanno resistenza e a loro volta la suscitano nella lingua, nei singoli parlanti, tanto che fino a non molto tempo fa si poteva distinguere il mondo fra tecnofili e tecnofobi. Oggi invece questa distinzione passa fra tecnofoni e tecnoanalfabeti, cioè tra quelli che sanno quali nomi dare 
alle trasformazioni quotidiane della nostra vita e quelli che le subiscono, o le comprano e le usano, senza riuscire a dirle.

\section{Le parole chiave del linguaggio tecnologico}

Uno dei motivi della difficoltà del linguaggio tecnologico a inserirsi nella lingua come un corpo vivo e attivo sta proprio nel fatto che la tecnologia e il linguaggio sono come due nemici. O meglio dire che la tecnologia è un linguaggio a parte che ha sempre cercato di sostituire le parole del linguaggio comune con le proprie parole e con un proprio ordine del discorso. Cominciamo dall'aggettivo digitale che ha modificato l'epoca stessa in cui viviamo e che rimanda a una vera rivoluzione linguistica (Granieri 45). II vecchio linguaggio analogico è stato sostituito con un altro puramente numerico e in tal modo è scomparso il sistema dei segni allusivi che riproducono in qualche modo le caratteristiche dell'oggetto rappresentato (ad esempio la posizione delle lancette dell'orologio come significante di una variazione temporale). Si è passati così dai robot che simulano il funzionamento del cervello agli avatar che riproducono la gestualità umana.

Oltre a questo l'essere umano è diventato il territorio privilegiato sul quale si dibatte il dilemma sulla crescente richiesta di sicurezza e di controllo e sul diritto alla salvaguardia della privacy. Resta importante notare che per i "nativi digitali", il digitale diventa la norma, come per le generazioni precedenti lo è stato il telefono e il televisore. Per i nati negli anni Ottanta del secolo scorso o dopo sarà perfino difficile ricordare un mondo in cui i network non erano così pervasivi (Granieri 39).

Con il progresso tecnologico nascono inoltre il sostantivo la biometria e l'aggettivo biometrico (passaporto biometrico) ${ }^{1}$, riferito all'utilizzo dei sistemi elettronici per identificare ogni individuo a partire da ciò che ha di unico, sia la voce o il Dna. Questo tipo di sperimentazione tecnologica ha aperto la strada alla nascita dell'uomo modificato e dell'uomo monitorato, più precisamente con il riferimento al braccialetto elettronico proposto per la sorveglianza dei detenuti. Intanto si procede mescolando vecchio e nuovo: la calligrafia è stata sostituita con la firma digitale con lo scopo di offrire maggiori garanzie contro le falsificazioni, mentre la carta d'identità elettronica e la chip card non si limitano a garantire la sicurezza ma diventano anche

\footnotetext{
1 "Passaporto dotato di un microcircuito digitale contenente dati relativi alle caratteristiche fisiche del titolare. [...] Composto dal sostantivo maschile passaporto e dall'aggettivo biometrico, ricalcando l'espressione inglese biometric passport." (Cfr. Adamo, Della Valle 2009, 489).
} 
multifunzionali. La loro multifunzionalità riunisce in un microprocessore i dati anagrafici, sanitari, genetici, nonché la possibilità di accedere alla rete, ai servizi bancari e amministrativi. Ancora qualche anno e si potrà, probabilmente, fare a meno di bancomat e carta di credito, gli acquisti si pagheranno con il documento d'identità.

La multifunzionalità (dall'inglese multitasking) è un campione particolarmente rappresentativo di quelli che Umberto Eco ha definito "termini feticcio". Forse uno dei più eclatanti, perché il suo significato vale anche come definizione delle loro caratteristiche e del loro comportamento linguistico: termini specifici di una disciplina che vanno oltre i loro limiti semantici originari e finiscono col riferirsi a oggetti diversi, in ambiti disciplinari anche distanti, diventano così parole multifunzione (Roncaglia 2012, 264-265).

Oltre a prendere le parole dagli altri linguaggi (ad esempio clonare prestato dalla medicina all'informatica e alla telefonia), la tecnologia molto più spesso ha dato, colorando la lingua dell'uso di singole parole oppure di locuzioni usate in senso figurato: bypassare per 'aggirare un ostacolo', monitorare per 'controllare', interfacciare per 'stabilire un contatto', mettere il turbo per 'accelerare', staccare la spina per 'interrompere ogni attività'.

Ci sono invece altre parole che hanno compiuto il percorso opposto, partendo da un significato molto ampio per specializzarsi in un ambito semantico preciso e invadere poi, in quella specifica accezione, vari settori: è il caso dell'aggettivo intelligente e del suo corrispettivo inglese smart (Adamo, Della Valle 2009, 570-571). Si tratta dell'aggettivo che si riferisce al significato di 'regolato da un computer' e che accompagna diversi concetti del vivere contemporaneo dai bisturi smart o bisturi intelligenti agli alberghi smart o alberghi intelligenti, dalle case intelligenti alle carte intelligenti. La tecnologia in questo modo sta imitando l'uomo cercando quasi di renderlo superfluo. La velocità con cui si susseguono i cambiamenti e i progressi della tecnologia si potrebbe presentare attraverso il cambiamento del computer, l'ormai familiare pc. Nel corso di qualche decennio il desktop da scrivania si è evoluto in computer rimpicciolito per spostarsi infine sullo schermo del televisore e soprattutto sul telefonino, grazie alle memory card (scheda di memoria) ${ }^{2}$ che trasferiscono memoria da un apparecchio all'altro.

\footnotetext{
2 "Scheda di memoria, supporto magnetico per l'immagazzinamento dei dati di un dispositivo digitale (computer, fotocamera, telefono cellulare, ecc.)." Cfr. Lo Zingarelli 2020.
} 


\section{I termini della telefonia fissa e mobile}

I requisiti delle nuove macchine e i termini che indicano l'orizzonte privilegiato della tecnologia moderna sono, senz'altro, multifunzione, compatibilità e interconnessione. Si tratta della possibilità di far comunicare strumenti diversi o di racchiudere in un unico strumento funzioni e prestazioni finora disperse in più apparecchi. Così il wireless ('senza fili') ha abolito i cavi che collegano i singoli apparecchi. La tecnologia bluetooth, che utilizza le onde radio, ne ha ampliato il raggio d'azione arrivando a mettere in contatto più apparecchi. Ma l'ambito in cui l'influenza tecnologica è palpabile al massimo è quello della telefonia fissa e mobile. Si potrebbe notare che la stessa distinzione che ha reso l'apparecchio di casa o dell'ufficio semplicemente 'il fisso', passa attraverso la presenza o meno dei fili. A dire il vero, il primo -less, ovvero la capacità di fare 'senza', è stato il cordless, il telefono fisso che fisso non era più visto che si poteva usare percorrendo tutta la casa.

Dall'altra parte, la telefonia mobile, invece, è mobile davvero. A partire dai primi cellulari, per i quali il termine telefonino era più vezzeggiativo che descrittivo, visto che erano voluminosi come citofoni, si è arrivati ai moderni microscopici apparecchi in cui sembra si voglia concentrare la pluralità delle tipologie di comunicazione come internet, musica, fotografia, video. Ma il mutamento più radicale sta nel fatto che mentre il telefono di casa corrisponde a un luogo, il telefonino corrisponde a una persona. L'identificazione dell'utente tramite il telefono ha lasciato il posto all'identificazione dell'utente con il telefono e non a caso ogni cellulare è blindato da PIN e sim card, codici segreti preziosi quanto quelli delle carte di credito e che, come i processori del computer, contano più del rivestimento che li nasconde. Grazie alla sim card, il numero di telefono di un cellulare è diventato portabile e la portabilità del numero permette a un utente di coincidere con il suo recapito telefonico mobile, sia che passi da un fornitore all'altro, sia che viaggi per il mondo, grazie agli accordi di roaming tra le varie compagnie internazionali. I telefonini di nuova generazione hanno una sorta di GPS (global positioning system) incorporato che permette ai genitori di sapere sempre dove si trovano i figli. Dall'altra parte, è anche ben noto, che il cellulare è di per sé un GPS, come dimostrano ogni giorno i servizi di intelligence quando rintracciano i nascondigli di terroristi seguendo i segnali dei loro apparecchi. Va detto che la rivoluzione telefonica ha trasformato ogni singolo utente in un ufficio ambulante e succede che oggi ognuno di noi porta con sé il luogo nel quale può essere rintracciato. 


\section{La lingua della telefonia mobile e degli sms}

Oltre ad essere una società tecnologica la nostra è anche la società delle immagini e per questo anche la telefonia mobile ha aggiunto alla comunicazione verbale un tipo di comunicazione figurale, sia in senso strettamente visivo, che in quello della manipolazione grafica della lingua. Gli short message, sono stati tradotti in italiano come messaggini, sempre all'insegna dei diminutivi che dominano nel linguaggio tecnologico. Come si sa, si tratta dei brevissimi testi scritti di non più di 160 caratteri da inviare e ricevere sul cellulare e che hanno dato alla lingua un contributo di parola tecnica messaggistica e un altro di parola gergale messaggiare. Va detto inoltre che la comunicazione mobile ha toccato e cambiato piuttosto le norme della lingua scritta. Così come per le e-mail, esiste una lingua degli SMS ed è un linguaggio che rispetta le regole della velocità e della sintesi. Una lingua che non è una lingua, semmai una diversa trascrizione fonetica di parole: ad esempio "ke" al posto di "che" o gruppi di parole dell'uso quotidiano, eppoi qualche contrazione ("cmq" per "comunque"), una serie di sigle ("tvb" per "ti voglio bene"), alcune combinazioni alfanumeriche che fondono simboli o numeri, la cui pronuncia corrisponde alle lettere 'economizzate' (" 6 " per indicare la seconda persona singolare del verbo essere, "3-do" per "tremendo", "4ever" per "forever") e il fantasioso riciclaggio di simboli matematici ("xò" per "però", "xché" invece di "perché", "+ o -“ al posto di "più o meno"). Molte di queste abbreviazioni si trovano più in generale nella scrittura giovanile e vengono mutuate dalla lingua delle chat ("msg" per "messaggio") (Fiorentino 2010, 199). Del resto, i giovani d'oggi sono già nati con internet, e considerano spesso il computer e la tecnologia alla stregua di qualsiasi altro elettrodomestico (Gheno 175).

Però con il progressivo invecchiare dei nuovi media è stato rapidissimo anche il declino di quel vecchio slang grafico e gli sms sono ormai in procinto di scomparire, rimpiazzati dai vari servizi di messaggeria gratuita (Serianni 17). II paradigma digitale viene seguito dal paradigma multimediale perché al telefonino si è presto sostituito lo smartphone la cui caratteristica principale è soprattutto l'integrazione con le altre forme di comunicazione (foto, video, audio), che spesso riduce la parola scritta a un ruolo accessorio (Serianni 13-14).

Dalle chat vengono mutuati anche le emoticon, ossia le faccine ottenute con caratteri della tastiera per aggiungere chiavi di interpretazione alle frasi (faccine che sorridono, faccine tristi, ammiccanti) (Fiorentino 2004, 78-79) e di recente alle emoticon si preferiscono di gran lunga gli emoji: quei disegnini fatti per 
rappresentare stati d'animo e oggetti vari. ${ }^{3}$ Pare che l'italiano si stia adattando all'ultimo salto tecnologico con tutta la vitalità e la flessibilità tipiche di una grande lingua nazionale (Lorenzetti e Schirru 71).

La rivoluzione telefonica non si è fermata al lessico, ma ha avuto conseguenze anche sulla sintassi dell'italiano parlato. Una di queste è il costrutto "sul telefonino", usato più frequentemente dell'atteso "al telefonino" per la sostituzione del quale, secondo Fabio Rossi ci sono tre spiegazioni: "l'influsso dall'inglese ("to call someone on the phone"), la maggiore diffusione di "su" rispetto ad "a" o "in" per molti costrutti locativi dell'italiano recente, e, infine, il fatto che "il telefonino è percepito quasi come un'appendice del corpo di chi lo possiede. La preposizione su, più di $a$, sembra rimandare a questa estrema vicinanza del telefono al suo possessore e per quello gli amici si chiamano sul cellulare" (94).

Il telefono, combinandosi con il computer, ha prodotto la società telematica e ha cambiato le nostre abitudini. Da qui la nascita dei call center che ci promettono la soluzione rapida di qualsiasi problema, senza doverci muovere da casa, col risultato di tenere tante persone attaccate alla cornetta ad ascoltare la voce interattiva ('interactive voice response). Si deve però notare che l'importanza della comunicazione mediata in rete ha dato, secondo molti osservatori, un'immediatezza nuova alla scrittura che si è dotata di alcune funzioni informali, in precedenza svolte solo dal parlato (Tavosanis 93). Secondo Luca Serianni l'era digitale è stata quella del ritorno alla scrittura e nel giro di pochi anni, siamo diventati tutti graforroici: blog, chat, e-mail e soprattutto messaggini (13).

\section{Le parole dell'industria dell'intrattenimento}

Negli ultimi decenni la tecnologia indirizzata e applicata alla casa ha cambiato profondamente il nostro ambito dei consumi culturali. Si tratta di quel vasto settore chiamato entertainment (intrattenimento), una locuzione ibrida che ha allontanato da noi l'era delle tombole d'una volta. Diverse attività ricreative tra le pareti di casa sono affidate a strumenti sempre più sofisticati, capaci di trasformare il salotto in una sala da concerto o in un cinema su misura. L'home

\footnotetext{
${ }^{3}$ Le emoticon erano apparse negli anni Ottanta per accompagnare le parole con una sorta di punteggiatura mimica. Gli emoji, inventati in Giappone negli anni Novanta, puntano invece a sostituire le parole, creando un nuovo esperanto. A partire da un'idea di Francesca Chiusaroli, Johanna Monti e Federico Sangati, un lavoro di gruppo creato su Twitter ha dato vita a un Pinocchio in Emojitaliano, apice libri, Firenze, 2017. (Cfr. Serianni. 2019, 17-18).
} 
theatre promette proprio questo: fare di ogni abitazione un teatro, dove godersi il film in DVD su un televisore al plasma o a cristalli liquidi, in formato widescreen, con la qualità visiva della retroproiezione e le suggestioni sonore del surround (Adamo, Della Valle 2003, 28, 57). Succede che, chiuso in casa tra gli innumerevoli giocattoli che l'industria dell'intrattenimento propone, l'uomo tecnologico può non solo fare a meno di uscire alla ricerca di occasioni di divertimento, ma diventare egli stesso il produttore. Così, accanto ai semplici duplicatori che sfruttano le nuove tecnologie e masterizzano la musica e i film, appaiono infatti i fotoamatori e i registi della domenica, muniti di fotocamere e videocamere digitali. Si formano in tal modo le parole come scanditi, scannerizzati o scansionati i cui risultati viaggiano via internet o passano per un masterizzatore.

Una volta si riteneva che i videogame fossero un divertimento per adolescenti. Ora non più. Oggi sono diffusi in tutto il mondo e ci giocano tutti: dai bambini agli adulti, l'età media del giocatore si aggira sui 30 anni, e il genere è equamente diviso tra uomini e donne (Granieri 57). Proprio nei nomi di due videogiochi più popolari si riesce a capire la potenza dei marchi commerciali, la playstation e il gameboy, sono infatti nomi commerciali registrati dai vocabolari ed elevati al rango di nomi comuni, grazie al numero di vendite di questi prodotti di fama internazionale. ${ }^{4}$ Nella versione playstation, che trasforma il televisore in una lanterna magica interattiva, utilizzando i comandi di un piccolo computer (console) e muovendosi in quello spazio virtuale con un joystick, i videogiochi possono essere considerati una forma di intrattenimento domestico. Nella versione gameboy, piccoli schermi portatili delle dimensioni di un telecomando, somigliano piuttosto a dei telefonini.

Bisogna sottolineare che il fattore gioco per il digitale è sempre stato importantissimo e molte persone, soprattutto i ragazzini, si sono avvicinati ai computer e ai dispositivi digitali, in generale, attraverso il gioco. Perciò il gioco non va sottovalutato né come dimensione di apprendimento né come una soluzione per superare le barriere cognitive che a molte persone la tecnologia ancora impone (Granieri 56).

\section{Le parole del progresso nell'industria automobilistica}

Nell'ambito ristretto della vita quotidiana sembra che il progresso tecnologico voglia dispensarci dalla fatica di premere un tasto o uscire di casa per andare al cinema o a un concerto. Anche le

\footnotetext{
4 “Playstation ${ }^{\circledR}$, s. f. inv. voce inglese, composta di play 'gioco' e station 'stazione', quadro di comando per computer game”. Cfr. Lo Zingarelli 2020.
} 
automobili, forse più delle case si avviano a fare tutto da sole, con la partecipazione minima del guidatore, che in certi casi è esentato perfino dallo sforzo di avviare il motore: basta che la macchina sia munita di un dispositivo easy go.

Dall'altra parte lo sforzo che la tecnologia si propone di risparmiarci non è soltanto quello di usare, riparare o sostituire gli strumenti che ci assistono, ma anche quello di saperne il nome. II linguaggio tecnologico è soprattutto quello della massima sintesi, le sigle rappresentano la base del suo lessico e il cui significato esteso non ha nessuna importanza conoscere. Basta che ad ogni insieme di vocali e consonanti corrisponda un concetto immediatamente visualizzabile, ad esempio la frenata automatica per l'ABS. L'obiettivo è trasformare le macchine in centrali operative al servizio del comfort, della sicurezza e ultimamente anche al servizio della fretta di chi le occupa (si pensa al telepass per saltare le code al casello).

Così si parla del navigatore interattivo e dei sistemi di navigazione satellitare che servono ad informare gli automobilisti non solo sul percorso più breve ma anche sulle condizioni meteorologiche $\mathrm{e}$ sulla lunghezza delle file ai semafori. Va detto che l'industria automobilistica è diventata un vero oggetto di sperimentazione. Sul piano linguistico "auto" si rivela un morfema usato indifferentemente come prefissoide o suffissoide ma quasi esclusivamente nella traduzione inglese (car) per definire vari tipi di automobile: minicar, microcar, citycar. Da una parte, il problema del parcheggio ha fatto nascere sia la nuova terminologia che i nuovi tipi di macchine che girano intorno ai prefissi diminutivi mini o micro. E dall'altra parte il problema dell'inquinamento ha contribuito invece ad accelerare la ricerca su forme alternative alla benzina. Si parla così dell'auto elettrica, guardata ancora con diffidenza a causa della scarsità delle colonnine di ricarica disponibili; dell'auto ibrida i cui due motori funzionano rispettivamente a elettricità e a benzina e infine dell'auto solare, dotata di motore alimentato con energia solare (Adamo, Della Valle 2009, 8488).

\section{Dalla rivoluzione tecnologica alla rivoluzione linguistica e lessicale}

La rivoluzione tecnologica non ha trasformato soltanto gli oggetti che ci circondano ma ha portato nella lingua una metamorfosi analoga. Perché il primo problema che si pone davanti a un fenomeno nuovo non è cosa farne, ma come dirlo. Nell'italiano, che prima di adattarsi al mondo digitale ha mutuato dall'inglese la maggior parte dei termini legati alla tecnologia, permangono ancora molte oscillazioni 
terminologiche. Secondo le parole di Gino Roncaglia "non si tratta semplicemente di analizzare prestiti e neologismi di un settore 'alla moda', ma di comprendere il funzionamento e i cambiamenti della lingua nel suo incontro con un ambiente comunicativo nuovo" (2010, 210). Tuttavia gli aspetti più eclatanti della rivoluzione linguistica che ha fatto seguito alla rivoluzione tecnologica sono: l'uso del diminutivo, il trionfo dell'acronimo e l'innata concisione dell'inglese, lingua ufficiale della tecnologia. In ogni caso il fenomeno ha creato molte parole nuove. Grazie al materiale raccolto, tratto da fonti giornalistiche e lessicografiche, nell'analisi si sono rivelate numerose parole nuove che da qualche decennio abbondano nel linguaggio della tecnologia e che in questa ricerca abbiamo tentato di presentare attraverso la riflessione con la quale si è cercato di cogliere in che modo e per quali strade $i$ cambiamenti della tecnologia partecipino a creare parole ed espressioni nuove. Riportiamo per di più una lista con le 110 parole ed espressioni, dalle rubriche di attualità di due quotidiani nazionali italiani, la Repubblica e La Stampa, pubblicati nel periodo dal 1응 ottobre al 31 dicembre del 2016. Alla lista aggiungiamo 15 sigle che hanno assunto un'autonomia di significato a livello globale, indipendentemente dalla capacità di chi li utilizza di scioglierne la sigla. Quindi, si tratta, in tutto, dei 125 neologismi che nel corpus studiato risaltano, dato che appaiono con più di 3 occorrenze, però molti dei quali non hanno ancora trovato il loro posto nei più importanti vocabolari italiani. Ma poiché oltre al campo semantico della tecnologia in generale, emerge un altro campo che si può individuare come il campo semantico della rete dove internet viene rappresentato non solo come una rete ma anche come un mare, si potrebbe proporre un'altra lista di termini informatici che in questa ricerca non sono stati presi in considerazione, a partire da navigare, navigatore, navigazione, per arrivare a surfer, surfista, surfing e ai due prefissi molto produttivi di questo campo semantico: cyber- (cyberspazio, cybermodo) o $e-(e-$ world). ${ }^{5}$

\footnotetext{
${ }^{5}$ Per dirla con Giovanni Adamo e Valeria Della Valle, da alcuni anni assistiamo a una progressiva sostituzione e affermazione di elementi formanti veicolanti in ambito mondiale dalla lingua inglese, o angloamericana. Sono noti a tutti alcuni esempi molto produttivi: cyber-, e-, info-, net-, web. (Cfr. Adamo, Della Valle2003, XVII).
} 


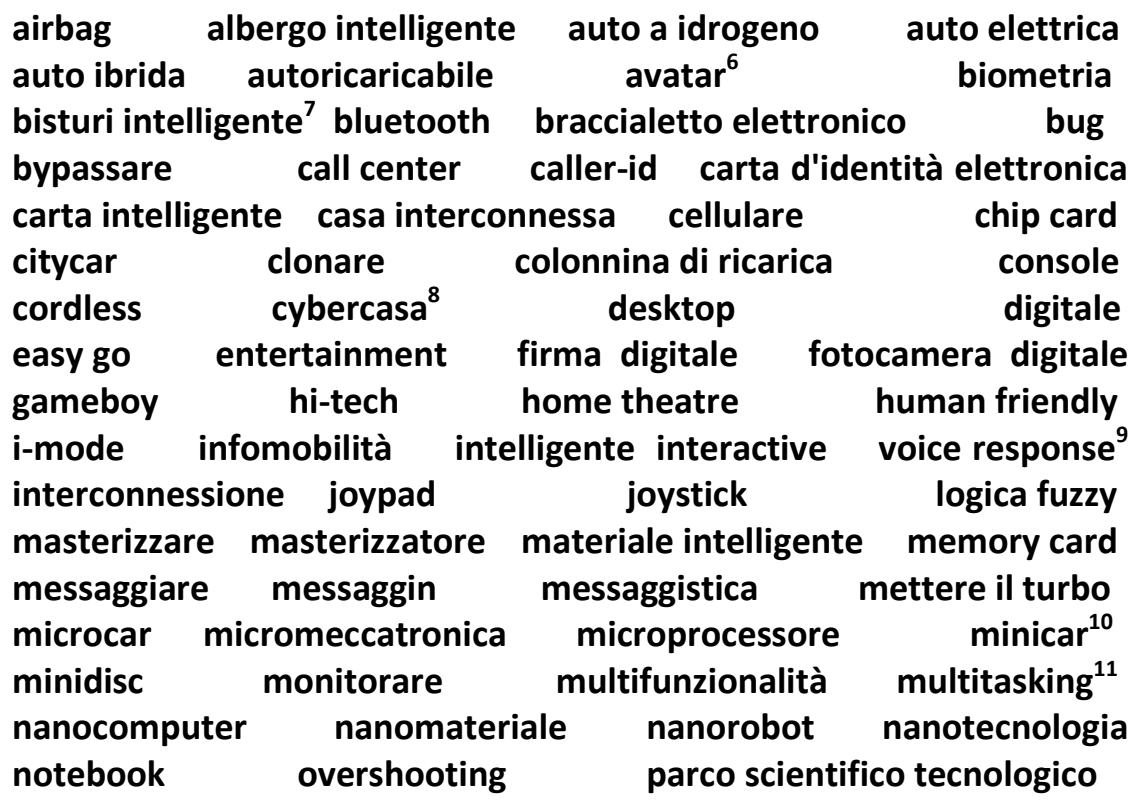

6 "Tra le mille invenzioni in corso, l'attenzione è puntata soprattutto sui cosiddetti Avatar, figure virtuali che parlano e si muovono, potenziali ottimi speaker per la tv. Questi visi hanno una mobilità eccelsa e i loro movimenti della faccia sono perfettamente sincronizzati con i fonemi, tanto ben costruiti che anche i sordomuti, attraverso il labiale, possono comprendere le singole parole." la Repubblica, 21 ottobre 2016.

7 "Scansa le cellule sane, scopre quelle malate, guida la mano del chirurgo durante gli interventi più delicati. È il bisturi del futuro, un sistema tecnologico avanzato in grado di selezionare le superfici da incidere, il sistema del bisturi intelligente." la Repubblica, 10 ottobre 2016.

8 "L'abitazione del futuro si gestirà senza chiavi ma grazie a telefonini." La Stampa, 25 ottobre 2016.

${ }^{9}$ "Ci sono i call center delle grandi aziende, che impiegano i numeri verdi per corteggiare i clienti. [...] L'informatizzazione consente un aumento del numero delle chiamate grazie all'interactive voice response. È quel sistema che quando chiediamo l'orario di un treno ci fa sillabare il nome della stazione desiderata." La Stampa, 13 novembre 2016.

10 "Il meglio della minicar resta il suo stesso concetto: facile e comoda come un'auto. L'ideale per andare al cinema o a fare la spesa anche quando diluvia, si trova posto davvero dappertutto." la Repubblica, 17 dicembre 2016.

11 "Multitasking: un talento al femminile. Di che cosa si tratta? È l'abilità nello svolgere più compiti contemporaneamente. Abilità nella quale le donne eccellono [...] Non deve stupirci che nascano, proprio per loro, specialità multitasking, efficaci in più direzioni: abbronzano e trattano la cellulite, proteggono dai danni solari e rassodano i tessuti." la Repubblica, 14 novembre 2016. 


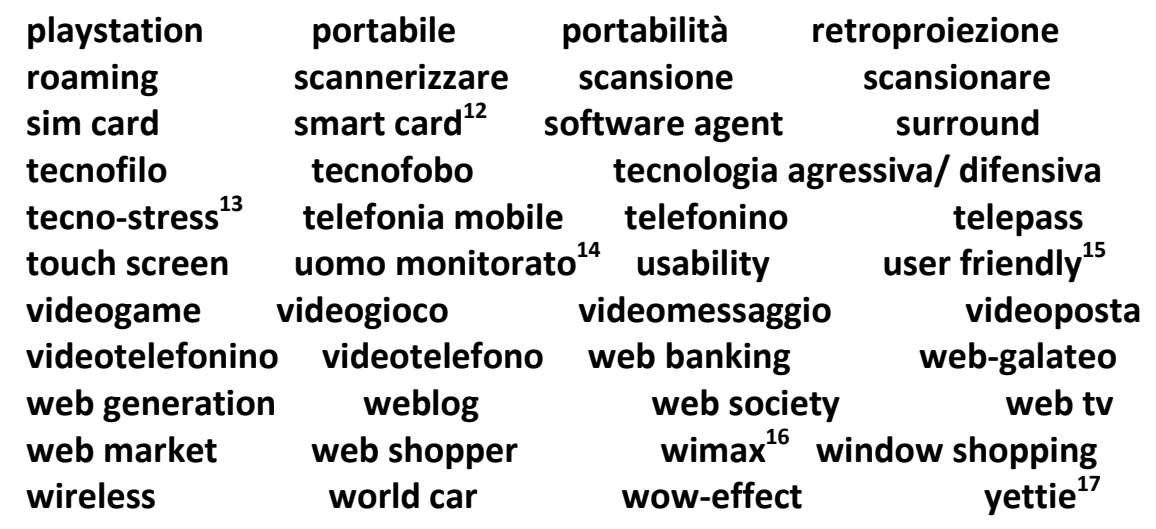

ABS ADSL CD-rom DVD DVD-ram DVD-rom GPS GSM MMS PIN PUK SMS WAP (Wireless Application Protocol) Wi-Fi (Wireless Fidelity) WWW (World Wide Web)

\footnotetext{
12 "Sotto la loro carrozzeria di plastica batte un cuore di silicio che è un vero e proprio microcomputer e che trasforma una semplice tessera in una carta intelligente (una smart card). II loro chip può non solo memorizzare una grande quantità di dati, ma anche elaborarli e scambiarli con altri dispositivi. La Stampa, 16 ottobre 2016.

13 "Mobbing, in banca esplode il tecno stress. Una forma di reazione generalizzata di adattamento dell'organismo, di stress, in cui gli stimoli che provengono da ambienti con alta tecnologia vengono elaborati in maniera patologica con conseguenti comportamenti di disadattamento." la Repubblica, 17 dicembre 2016.

14 “L'uomo sta diventando una password, dove la fisicità prende il posto di astratte parole-chiave sostituite da impronte digitali, geometria della mano, delle dita o dell'orecchio. Da iride, tratti del volto, odore, voce, andatura. [...] Uomo monitorato, uomo modificato, al guinzaglio elettronico." la Repubblica, 29 ottobre 2016.

15 “Da noi i quotidiani e le riviste perdono copie. Tra i giovani sotto i 25 anni quasi nessuno, meno di uno su cinque, segue il nostro lavoro. Fanno bene. Non siamo, come si dice nel mondo in crescita dei computer, user friendly amici di chi ci usa, ignoriamo i loro interessi a meno che non si vadano a spiaccicare contro un albero ritornando dalla discoteca." la Repubblica, 23 ottobre 2016.

16 "Wimax: potenziamento del wi-fi. Connette case e edifici non raggiungibili dai cavi." la Repubblica, 13 dicembre 2016.

17 "Sono giovani e veloci. Informati e informali. Internet è il loro rock'n roll, il pc la loro chitarra. Mancava loro soltanto una cosa, piccola ma decisiva, un nome. Ora ce l'hanno: yetties. Y come young (giovani), E come enterpreneurial (imprenditoriali), T come tech-based (tecnologicamente avanzati)." la Repubblica, 15 dicembre 2016.
} 
Dalla panoramica delle parole proposte risulta evidente la forte presenza della lingua inglese nella formazione delle nuove parole della tecnologia. Oltre alle 15 sigle di provenienza e forma puramente inglese, tra le 110 parole ed espressioni persino 56 termini sono inglesi, diffusisi a livello globale e riconoscibili alla perfezione a un utente con la minima padronanza del mondo tecnologico. Invece, soprattutto per l'esigenza di coniare una nuova parola, gli altri 54 termini tra parole ed espressioni proposte sono stati tradotti o adattati al bisogno di un parlante italiano (carta intelligente, casa interconnessa, firma digitale, messaggiare, portabilità, telefonia mobile ecc.). In altre parole, il 57\% dei termini trattati risultano di matrice inglese, mentre il $43 \%$ dei termini sono neologismi italiani che rispettano le regole di formazione dell'italiano e utilizzano gli elementi già presenti nella lingua. Di coniazione inglese o italiana, si tratta delle parole che entrano nella comunicazione corrente in maniera tanto capillare da diventare patrimonio lessicale di tutti.

Dato che non c'è più tempo per opporsi al progresso tecnologico e nemmeno ai termini tecnologici, siamo ben consapevoli del fatto che la lingua italiana presenta le stesse perplessità e gli stessi timori linguistici che vivono anche altre lingue. Inoltre il progresso corre veloce, più veloce della nostra capacità di adeguamento. E si capisce allora come si può andare addirittura in 'overdose da eccesso di informazioni e di innovazioni' (overshooting e overburning), allo stesso modo in cui una macchina va in tilt, o come si possono accusare i sintomi di tecno-stress, nonostante tutte le promesse di 'amichevolezza e facilità' delle novità della tecnica, definite di volta in volta user friendly, human friendly, easy use e plug and play, ovvero 'inserisci la spina e gioca', d'altronde come tante altre locuzioni ricalcate sull'inglese, del tipo "gratta e vinci" e "mordi e fuggi". Probabilmente, ogni volta che incontriamo un nuovo fenomeno o oggetto tecnologico dovremmo ricercarne la bellezza dentro queste nuove possibilità linguistiche e lessicali che permettono di capirci globalmente senza dover ricorrere all'uso di internet o di un dizionario di neologismi.

\section{Note conclusive}

Come tutte le rivoluzioni anche quella tecnologica rispecchiatasi nella lingua ha scatenato diverse opinioni, tra quelli che sono a favore delle novità e quelli che ne sono contrari. Non è solo un tema di moda, ma quasi un'ossessione che coinvolge soprattutto linguisti e giornalisti. Il confine di questa moderna divisione va ancora più avanti e distingue gli alfabeti e gli analfabeti della tecnologia, separa 
anche le generazioni e ne definisce una in particolare, quella di chi ha giocato prima con il mouse e telefonino che con il trenino e la Barbie.

Come abbiamo visto dalle neoformazioni nel linguaggio della tecnologia succede che la lingua accetta e adopera i termini stranieri, oppure li adatta al proprio sistema lessicale. Ci riferiamo alla diffusione di nuove forme, di veri e propri internazionalismi che si adattano o ricalcano in vario modo, elementi lessicali di provenienza inglese o angloamericana come in tanti altri settori. Inoltre, come è già stato notato, le nuove modalità testuali e le nuove tecniche conversazionali, che suonano anomale alle nostre orecchie, sono dovute maggiormente ai nuovi sistemi di comunicazione (sms, e-mail, chat-line) e molto spesso dipendono dalla voluta informalità della situazione. Quello che emerge non è altro che l'immagine di un preciso momento storico e sociale della lingua e della cultura che essa esprime. L'origine è da ricercare nella velocità di una tecnologia che non conosce più frontiere e nel nostro modo di vivere immerso nel complesso fenomeno dello sviluppo tecnologico (Bralić 480). Da non scordare il fatto che le tecnologie assumono una particolare importanza come un valido apporto anche al processo di apprendimento perché il supporto dato dalle tecnologie digitali è significativamente spendibile nel panorama della scuola (Bruni, Fiorentino 13). Secondo Vera Gheno la sfida dei prossimi anni sarà quella di includere la tecnologia e la multimedialità tra le esperienze necessarie alla formazione del singolo (183).

\section{Bibliografia:}

Adamo, Giovanni e Valeria Della Valle. Neologismi. Parole nuove dai dizionari. Roma: Istituto della Enciclopedia Italiana Treccani, 2009.

Neologismi quotidiani. Un dizionario a cavallo del millennio 1998-2003. Firenze: Olschki editore, 2003.

Bralić, Snježana. "Globalizzarsi o morire: dal mondo globale al lessico globale". Letteratura, arte, cultura tra le due sponde dell'Adriatico ed oltre. A cura di Balić Nižić, Nedjeljka, Luciana Borsetto e Adrijana Jusup Magazin. Zadar: Sveučilište u Zadru, 2016. 465-482.

Bruni, Filippo e Giuliana Fiorentino. Didattica e tecnologie. Studi, percorsi e proposte. Roma: Carocci, 2013.

Fiorentino, Giuliana. "Forme di scrittura in rete: dal web 1.0 al web 2.0." Lingua e linguaggio dei media. A cura di Aprile, Marcello. Roma: Aracne, 2010. 193-206. 
"Scrittura elettronica: il caso della posta elettronica". Scrittura e nuovi media. A cura di Orletti, Franca. Roma: Carocci, 2004. 69-112.

Galli, Riccardo. Innovazione. Le parole della tecnologia. Roma: Ediesse, 2005.

Gheno, Vera. "I giovani e la comunicazione mediata dal computer: osservazioni linguistiche su nuove forme di alfabetizzazione". Verbum Analecta Neolatina XI/1 (2011):167-187.

Granieri, Giuseppe. Umanità accresciuta. Come la tecnologia ci sta cambiando. Roma-Bari: Laterza, 2009.

La Stampa. 1 ottobre 2016 - 31 dicembre. Torino: 2016.

La Repubblica. 1 ottobre 2016 - 31 dicembre. Milano: 2016.

Lorenzetti, Luca e Giancarlo Schirru. "La lingua italiana nei nuovi mezzi di comunicazione: SMS, posta elettronica e Internet". Guida alle pratiche della comunicazione. A cura di Gensini, Stefano. Roma: Carocci, 2006. 71-89.

Roncaglia, Gino. "Il topo scannato. Italiano e terminologia informatica". Lingua italiana e scienze. A cura di Nesi, Annalisa e Domenico De Martino. Firenze: Accademia della Crusca, 2012. 261-269. "Linguaggi e tecnologia: usi della lingua e strumenti di rete". Libro dell'Anno 2010. Roma: Istituto dell'Enciclopedia Italiana Treccani, 2010. 207-320.

Rossi, Fabio. "Chiamare sul cellulare". Studi linguistici italiani XXVII (2001): 93-96.

Serianni, Luca. L'italiano, parlare, scrivere, digitare. Roma: Treccani, 2019.

Tavosanis, Mirko. L'italiano del web. Roma: Carocci editore, 2012 [2011].

Zingarelli, Nicola. Lo Zingarelli 2020. Vocabolario della lingua italiana. Bologna: Zanichelli, 2019.

\section{KLJUČNE RIJEČI TEHNOLOŠKE REVOLUCIJE I NOVE LEKSIČKE TENDENCIJE}

Imajući u vidu da je tehnološki napredak zahvatio sva područja našeg života, ovaj rad pridonijet će razumijevanju činjenice koja govori o tome kako tehnološke inovacije i jezik kojim se služe nisu više specijalistički govori struke, već svakodnevni, suvremeni način izričaja. U radu se ne promišlja isključivo lingvistički, a novi termini i izrazi koji su nastali tvorbom kao rezultat napretka i tehnološke revolucije predstavljaju leksičko bogatstvo talijanskog jezika te su preuzeti iz dnevnih novina, 
leksikografskih izvora i s mrežnih stranica. Povezivanjem različitih uređaja, a zahvaljujući mogućnosti da jedan uređaj preuzme više funkcija koje su do sada bile podijeljene na niz uređaja, nastali su primarni termini kojima se opisuje moderna tehnologija: multifunkcionalnost, kompatibilnost i međupovezanost.

$\mathrm{U}$ talijanskom jeziku, koji je prije svoje prilagodbe digitalnom svijetu iz engleskog preuzeo većinu termina vezanih uz tehnologiju, postoje brojne terminološke nejasnoće. Po riječima Gina Roncaglie "ne radi se samo o proučavanju posuđenica i novotvorenica preuzetih iz nekog trenutno 'modernog' područja života, nego je riječ o razumijevanju jezika te shvaćanju na koji se način jezik ponaša te do kakvih promjena $\mathrm{u}$ jeziku dolazi onda kada se susretne $\mathrm{s}$ novim komunikacijskim okruženjem". Postavlja se pitanje govori li tehnologija, je li promijenila jezik ili je samo leksik popunila kraticama, aglicizmima, novim oblicima izričaja koji poštuju pravila brzine i sažetosti. Brojni tehnološki izrazi smatraju se odbojnima, izazivaju otpor kod govornika, u toj mjeri da se govori o razlici između onih koji su tehnološki pismeni i onih koji to nisu (tecnofoni i tecnoanalfabeti), tj. između onih koji znaju kojim imenom nazvati promjene koje se $u$ tehnologiji svakodnevno događaju $i$ onih koji se tim novitetima i promjenama služe bez da ih nazivaju pravim imenom. Ovdje mislimo na fenomen nastanka i širenja novih tvorbenih oblika s internacionalnim obilježjima koji se prilagođuju na različite načine i na različitim razinama, na leksičke elemente engleskog ili angloameričkog podrijetla, na polju tehnologije kao i u brojnim drugim područjima.

Ključne riječi: talijanski leksik, jezik digitalne komunikacije, tehnološki izričaj, leksička morfologija, novotvorenice, pomodne riječi, posuđenice, kratice, informatički termini 